\title{
ON QUASIPROJECTIVE COVERS
}

BY

THEODORE G. FATICONI

\begin{abstract}
The main results determine the Goldie dimension of superfluous submodules of semiprime left Goldie rings and apply this to the study of quasiprojective covers of torsion free modules. Conditions are given to guarantee that a quasiprojective cover of a torsion free module is an isomorphism. A class of nonperfect rings is given such that finitely generated singular modules possess quasiprojective covers.
\end{abstract}

Introduction. In their groundbreaking paper [20], Wu and Jans introduced the notions of a quasiprojective module and a quasiprojective cover. These generalized the classical concepts of a projective module and a projective cover (see [5]). Since its introduction, the study of quasiprojective modules has been pursued with some degree of success (see $[3,8,11,12,19])$. However, except for [12], which describes perfect rings in terms of quasiprojective covers, little attention has been given quasiprojective covers. They wait, as it were, for the study of quasiprojectives to bear sufficient fruit. The purpose of this paper is to examine the structure of quasiprojective covers over various rings. In fact, most of our results demonstrate that quasiprojective covers over certain rings are projective covers over quotients of these rings. Thus, an examination of projective covers and superfluous submodules of free modules is to be expected. Ultimately, we give sufficient conditions on a ring which guarantee every finitely generated left singular module possesses a quasiprojective cover (Corollary 3.4). Moreover, conditions on a semiprime left Goldie ring are given which imply a quasiprojective cover of a torsion free left module is an isomorphism (Corollary 3.16).

0. Definitions, notations and conventions. The word ring will mean an associative, not necessarily commutative, ring with unit. The word module will mean a unital module with scalars written on the left. The symbol $R$ will always be a ring and $M$ will always denote a left $R$-module. If $R$ is an order (left and right) in an Artinian quotient ring $Q$, a left $R$-module $M$ is said to be torsion free if the canonical map of $M$ into $Q \otimes_{R} M$ is a monomorphism. The kernel of this map is called the torsion submodule of $M$, and is denoted $t(M)$. Define an $R$-submodule $N$ of $M$ to be superfluous in $M(N \ll M)$ iff for $R$-submodules $X$ of $M, N+X=M$ implies $X=M$. The symbol $Q$ will always mean an Artinian ring.

Definition. Let $M$ and $N$ be a pair of left $R$-modules.

Received by the editors November 3, 1981.

1980 Mathematics Subject Classification. Primary 16A50; Secondary 13C10.

(C)1983 American Mathematical Society $0002-9947 / 82 / 0000-0738 / \$ 04.25$ 
(1) $M$ is projective relative to $N$ (or $M$ is $N$-projective), iff $\operatorname{Hom}_{R}(M$, ) is exact with respect to sequences of $R$-modules of the form

$$
0 \rightarrow X \rightarrow N \rightarrow Y \rightarrow 0 .
$$

(2) $M$ is called quasiprojective iff $M$ is $M$-projective.

(3) The exact sequence of $R$-modules $M \rightarrow N \rightarrow 0$ is a projective cover of $N$ over $R$ (or briefly, a projective cover), iff $M$ is a projective $R$-module and the kernel of the map $M \rightarrow N$ is superfluous in $M$.

(4) The exact sequence in (3) is a quasiprojective cover of $N$ over $R$ (or briefly, a quasiprojective cover), iff $M$ is a quasiprojective $R$-module, the kernel $k$ of the map $M \rightarrow N$ is superfluous in $M$, and for nonzero submodules $L$ of $k, M / L$ is not a quasiprojective $R$-module. This last condition guarantees a quasiprojective module is its own quasiprojective cover.

Throughout the text, $C$ denotes a Noetherian commutative ring where the localization of $C$ at the set of regular (i.e. nonzero divisors) elements of $C$ is a commutative Artinian ring. This localization will always be denoted by $K$. Because most of our results are trivial for the case $C=K$, it will always be assumed that $C$ has at least one ideal which is not an ideal of $K$.

For a ring $R$ and a nonzero module $M$, the following conventions hold: $M^{(a)}$ is the direct sum of $a$ copies of $M$ for cardinals $a$; for $x$ in $M, l(x)$ is the left annihilator of $x$ in $R ; C(R), J(R), N(R)$ denote respectively the center, Jacobson radical, and nilpotent radical of the ring $R$.

Define the $R$-rank of a left $R$-module $M(R-\operatorname{rank}(M))$ to be the length of the $Q / N(Q)$-module $Q / N(Q) \otimes_{R} M$. If the length is unbounded, we say that $M$ has infinite rank. By the exactness of $Q / N_{Q}(Q) \otimes_{R} \cdot R$-rank is additive over short exact sequences.

Two facts frequently used are:

(0.1) "If $N \ll M$, and $f: M \rightarrow L$ is an $R$-module map, then $f(N) \ll L$."

(0.2) "Let $P(M)$ be the class of modules $N$ such that $M$ is $N$-projective. Then $P(M)$ is closed under submodules, quotients and finite direct sums." (Proposition 16.12 of $[1]$.)

1. Superfluous submodules of free modules and projective covers over semiprime rings. Let $R$ be an order in a semisimple Artinian ring $Q$, and let $F$ be a free $R$-module. Let $L$ be a submodule of $F$. The aim of this section is to determine the rank of $L$ if $L$ is a superfluous submodule of $F$. This information is then applied to projective covers over $R$.

Recall that one version of Nakayama's Lemma states that finitely generated submodules of $J(R) F$ are superfluous in $F$. The following lemma shows the converse to be true.

LEMMA 1.1. Say $R$ is a prime ring (i.e. $Q$ is a simple ring). If $L \subset F$ and $L$ has infinite $R$-rank, then $L$ is not superfluous in $F$.

Proof. Assume that $L$ and $F$ have infinite $R$-rank. The objective is to construct an $M$ such that $M+L=F$ but $M \neq F$. 
By $(0.1)$, it suffices to assume that $F$ is free on a countable basis. Let $B$ denote this basis. Consider $L, F$ and $B$ as subsets of $\bigoplus_{b \in B} Q b=Q F$. Let $Q L$ be the $Q$-submodule of $Q F$ generated by $L$. Index $B$ with the positive integers.

Because $L$ has infinite rank, $L$ is not contained in $Q b_{1}$. Since $Q$ is simple, one may pick a summand of $Q L$ which is isomorphic to $Q$, and disjoint from $Q b_{1}$. Let $y_{1}$ generate this summand. Because $L$ is an essential submodule of $Q L$, there is a regular element $c_{1}$ of $R$ such that $c_{1} y_{1}$ is an element of $L$. Notice that $l\left(c_{1} y_{1}\right)=(0)$ since $Q c_{1} y_{1}=Q y_{1} \cong Q$.

Assume there are $y_{1}, \ldots, y_{n} \in L$ and regular $c_{1}, \ldots, c_{n} \in R$ with $Q y_{n} \cap\left[Q b_{1}\right.$ $\left.+\cdots+Q b_{n}+Q y_{1}+\cdots+Q y_{n-1}\right]=(0)$ and $Q y_{1} \oplus \cdots \oplus Q y_{n} \subset Q L$ a free $Q$-module on $\left(y_{1}, \ldots, y_{n}\right)$. Further assume that $c_{1} y_{1}, \ldots, c_{n} y_{n}$ are elements of $L$. Notice for $i=1, \ldots, n, l_{R}\left(c_{i} y_{i}\right) \subset l_{Q}\left(c_{i} y_{i}\right)=l_{Q}\left(c_{i} y_{i}\right)=(0)$.

Because $L$ has infinite rank, $Q L$ is not contained in $Q b_{1}+\cdots+Q b_{n+1}+Q y_{1}$ $+\cdots+Q y_{n}$. Since $Q$ is simple, one may choose a submodule of $Q L$ which is isomorphic to $Q$, and disjoint from $Q b_{1}+\cdots+Q b_{n+1}+Q y_{1}+\cdots+Q y_{n}$. Let $y_{n+1}$ be the generator of this free submodule of $Q L$. Because $L$ is an essential $R$-submodule of $Q L$, there is a regular element $c_{n+1}$ of $R$ such that $c_{n+1} y_{n+1} \in L$. Since $c_{n+1}$ is a unit of $Q, l_{R}\left(c_{n+1} y_{n+1}\right) \subset l_{Q}\left(y_{n+1}\right)=(0)$. Continue. To ease notation, let $x_{i}=c_{i} y_{i}$ for $i=1,2, \ldots$.

Let $M=R\left(b_{1}+x_{1}\right)+R\left(b_{2}+x_{2}\right)+\cdots$. Then $L+M=F$ since this sum contains $B$, the basis of $F$.

Claim. $M$ does not contain the element $b_{1}$. If so, then $b_{1}=r_{1}\left(b_{1}+x_{1}\right)$ $+\cdots+r_{n}\left(b_{n}+x_{n}\right)$ for some elements $r_{1}, \ldots, r_{n}$ of $R$. Assume that $n \neq 0$ is minimal. This will produce a contradiction since then $r_{n} x_{n}$ is an element of $\left[R b_{1}+\cdots+R b_{n}\right.$ $\left.+R x_{1}+\cdots+R x_{n-1}\right] \cap R x_{n}=(0)$. Thus $r_{n} x_{n}=0$ implying $r_{n}=0$ by our choice of $x_{n}$. This contradiction to the minimality of $n$ implies $M \neq F$ and this in turn implies $L$ is not a superfluous submodule of $F$.

Corollary 1.2. Let $R$ be semiprime (i.e. $Q$ is semisimple Artinian), and let $F$ be a free $R$-module. Let $L$ be a submodule of $F$. If $L$ is superfluous in $F$ then $L$ has finite $R$-rank. In particular, if $R$ is a Noetherian ring, then $L \ll F$ iff $L \subset J(R) F$ and $L$ is finitely generated by $R$.

Proof. By the Wedderburn Theorem, write $Q=Q_{1} \times \cdots \times Q_{t}$ where $Q_{i}$ is a simple Artinian ring for $i=1, \ldots, t$. Let $B$ be a basis for $F$ over $R$. For $R$-submodules $X$ of $Q F=\bigoplus_{b \in B} Q b$, let $Q X$ be the $Q$-submodule of $Q F$ generated by $X$. Also, let $X_{i}$ denote the canonical projection of $X$ into the free $Q_{i}$-module $\bigoplus_{b \in B} Q_{i} b \subset Q F$. Thus, it is easily verified that $R$ is a subring of $R_{1} \times \cdots \times R_{t}$, and for $R$-submodules $X$ of $Q F, X$ is a submodule of $X_{1} \oplus \cdots \oplus X_{t}$. Notice that $R_{i}$ is a prime ring for $i=1, \ldots, t$.

Say $L \ll F$. By $(0.1), L_{i} \ll F_{i}$ for $i=1, \ldots, t$. Finally, $F_{i}$ is a free $R_{i}$-module since $F_{i}$ is merely $\bigoplus_{b \in B} R_{i} b$. Hence, each $L_{i}$ has finite $R$-rank which implies $L$ has finite $R$-rank.

In particular, if $L \ll F$ and $L$ has finite rank, then $L$ is contained in a finitely generated free $R$-module. Because $R$ is Noetherian, $L$ is a finitely generated 
$R$-module. A short exercise places $L$ in $J(R) F$. The converse is Nakayama's Lemma, so the proof is complete.

Knowledge of the superfluous submodules of free modules permits one to prove the following results.

THEOREM 1.3. Let $R$ be semiprime, and $M$ a left $R$-module. If $M$ possesses a projective cover over $R$ then there are projective $R$-modules $F, F^{\prime}$ and a finitely generated module $M^{\prime}$ such that $M^{\prime}$ has a free cover over $R$ and $M \oplus F \cong M^{\prime} \oplus F^{\prime}$. In particular for Noetherian $R$, the torsion submodule of $M$ is finitely generated.

Proof. Let $P \stackrel{\pi}{\rightarrow} M \rightarrow 0$ be the projective cover of $M$. There is a projective $R$-module $F$ such that $P \oplus F$ is a free $R$-module. By 1.2, there are free submodules $F^{\prime \prime}$ and $F^{\prime}$ of $P \oplus F$ with $F^{\prime \prime}$ finitely generated, ker $\pi \subset F^{\prime \prime}$, and $F^{\prime \prime} \oplus F^{\prime}=P \oplus F$. Then

$$
M \oplus F \cong(P \oplus F) / \operatorname{ker} \pi \cong\left(F^{\prime \prime} \oplus F^{\prime}\right) / \operatorname{ker} \pi \cong F^{\prime \prime} / \operatorname{ker} \pi \oplus F^{\prime} .
$$

Setting $M^{\prime}=F^{\prime \prime} / \operatorname{ker} \pi$ produces the stated result since $\operatorname{ker} \pi \ll F^{\prime \prime}$.

In particular, if $R$ is Noetherian, then the torsion submodule of $M$ is a submodule of $M^{\prime}$. Thus $t(M)$ is a finitely generated $R$-module. This completes the proof.

THEOREM 1.4. Let $R$ be any ring such that $N(R)$ is T-nilpotent and $R / N(R)$ is a semiprime Goldie ring. Say $P \stackrel{\pi}{\rightarrow} M \rightarrow 0$ is a projective cover over $R$. Then $M$ has finite $R$-rank iff $M$ is a finitely generated $R$-module.

Proof. One induces a projective cover over $R / N(R)$ by defining

$$
P / N(R) P \stackrel{\bar{\pi}}{\rightarrow} M / N(R) M \rightarrow 0
$$

to be the sequence induced by $P \stackrel{\pi}{\rightarrow} M \rightarrow 0$. By $(0.1)$, ker $\bar{\pi}$ is a superfluous $R$-submodule of $P / N(R) P$. Since $R / N(R)$-submodules of $P / N(R) P$ are $R$-submodules, ker $\bar{\pi}$ is a superfluous $R / N(R)$-submodule of $P / N(R) P$. Because rank is additive over short exact sequences, $\operatorname{rank}(P)=\operatorname{rank}(\operatorname{ker} \bar{\pi})+\operatorname{rank}(M)$.

Assume that the $R$-rank of $M$ is a finite integer. By the equality above and 1.2, $\operatorname{rank}(P)$ is finite. Because $P / N(R) P$ is a projective $R / N(R)$-module, $P / N(R) P$ is a finitely generated $R$-module. Since $N(R)$ is $T$-nilpotent, $N(R) P \ll P$ as $R$-modules [5]. Thus, $P$ is a finitely generated projective $R$-module. Consequently, $M$ is a finitely generated $R$-module. The converse is clear, so the proof is complete.

Theorem 1.3 can be thought of as a reduction to the case of finitely generated projective covers. In fact, more is true.

COROllaRY 1.5. Let $R$ be an order in a semisimple ring $Q$. The following are equivalent:

(1) The torsion free $R$-modules with projective covers are the projective $R$-modules.

(2) The torsion free $R$-modules of finite rank are the finitely generated projective $R$-modules.

(3) The torsion free finitely generated $R$-modules with projective covers are the finitely generated projective $R$-modules. 
Proof. (1) $\Rightarrow(2) \Rightarrow(3)$ is clear.

(3) $\Rightarrow$ (1). Let $P \stackrel{\pi}{\rightarrow} M \rightarrow 0$ be a projective cover of a torsion free $R$-module. By 1.3 , $M$ is a summand of $M^{\prime} \oplus F^{\prime}$ where $F^{\prime}$ is a projective $R$-module, and $M^{\prime}$ is a finitely generated $R$-module with a free cover. The hypothesis shows $M^{\prime}$ is a projective $R$-module and this implies $M$ is a projective $R$-module.

Note. If $R$ is a semihereditary order in a semisimple Artinian ring $Q$, then $R$ satisfies 1.5(2). Therefore 1.5(1) holds as well. For hereditary orders, stronger statements are possible.

COROLLARY 1.6. Let $R$ be a semiprime ring. The following are equivalent:

(1) $R$ is a left hereditary ring.

(2) Submodules of projective left $R$-modules have projective covers.

(3) Submodules of projective $R$-modules are quasiprojective.

(4) For left ideals $I$ of $R$, and cardinals $c, I^{(c)}$ is a quasiprojective module.

Proof. Let $I$ be a left ideal of $R$ and let $I^{(c)}$ be the direct sum of countably many copies of $I$. Then $I^{(c)}$ is a submodule of the projective $R$-module $R^{(c)}$.

$1 \Rightarrow 2$. If $R$ is left hereditary, the result is clear since submodules of projective modules are projective.

$2 \Rightarrow 1$. If every submodule of a projective module has a projective cover, then $I^{(c)}$ has a projective cover $P_{0} \stackrel{\pi_{0}}{\rightarrow} I^{(c)} \rightarrow 0$. Also, $I$ has a projective cover $P \stackrel{\pi}{\rightarrow} I \rightarrow 0$.

Claim. It suffices to prove $P_{0} \cong P^{(c)}$. If so, then $\operatorname{ker} \pi_{0}=(\operatorname{ker} \pi)^{(c)}$ is a superfluous submodule of $P_{0}$. By 1.2 , ker $\pi_{0}$ has finite rank while $(\operatorname{ker} \pi)^{(c)}$ has infinite rank if $\operatorname{ker} \pi$ is nonzero. Thus ker $\pi$ has rank zero. Because ker $\pi$ is torsion free, $\operatorname{ker} \pi=(0)$. Our choice of $I$ was arbitrary, so $R$ is a left hereditary ring.

To show $P_{0} \cong P^{(c)}$, observe that by uniqueness of the projective cover $P \stackrel{\pi}{\rightarrow} I \rightarrow 0$, one constructs a chain of submodules of $P_{0}: P \subset P^{(2)} \subset \cdots \subset P_{0}, P^{(n)} \oplus X_{n}=P_{0}$. $\left(P_{0} \rightarrow I \rightarrow 0\right.$ is projection into the first coordinate of $I^{(c)}$ so $P_{0}=P \oplus X_{1}$. But $X_{1}$ may be mapped onto the second copy of $I$ in $I^{(c)}$ so $X_{1}=P \oplus X_{2}$. Continue.) Therefore, $P^{(c)}$ is a submodule of $P_{0}$. Furthermore, given an element $x$ of $I^{(c)}$, there is an integer $n$ such that $x$ is contained in the first $n$ copies of $I$ in $I^{(c)}$. By the construction of the chain, there is a $y$ in $P^{(n)} \subset P_{0}$ which maps onto $x$. Then $P^{(c)}+\operatorname{ker} \pi_{0}=P_{0}$. Therefore $P_{0}=P^{(c)}$ and by the claim, the proof is complete.

$1 \Rightarrow 3 \Rightarrow 4$ is clear.

$4 \Rightarrow 1$. Let $I$ and $I^{\prime}$ be left ideals of $R$ such that $I \oplus I^{\prime}$ is an essential left ideal of $R$. Then $I \oplus I^{\prime}$ contains a copy of $R$. ( $I \oplus I^{\prime}$ contains a regular element of $R$ by [6].) By hypothesis and (0.2), $I \oplus I^{\prime}$ is $R^{(c)}$-projective for every cardinal $c$. Thus $I \oplus I^{\prime}$ is a projective $R$-module. One easily follows.

COROllaRY 1.7. Let $R$ be an order in a semisimple ring $Q$. The following are equivalent:

(1) $R=Q$.

(2) $Q$ has a projective cover over $R$.

(3) Every torsion free left $R$-module has a projective cover over $R$. 
Proof. (1) $\Rightarrow(3) \Rightarrow(2)$ is clear. To prove (2) $\Rightarrow(1)$, let $P \rightarrow Q \rightarrow 0$ be the projective cover in (2). By 1.3, $Q$ is a summand of $M^{\prime} \oplus F^{\prime}$ where $F^{\prime}$ is a projective $R$-module and $M^{\prime}$ has a free cover over $R$. Then $Q=\left(Q \cap M^{\prime}\right) \oplus\left(Q \cap F^{\prime}\right)$ since $Q$ is an injective $R$-module. For the same reasons, $M^{\prime}=\left(Q \cap M^{\prime}\right) \oplus M^{\prime \prime}$ and $F^{\prime}=(Q$ $\left.\cap F^{\prime}\right) \oplus F^{\prime \prime}$ for some $R$-modules $M^{\prime \prime}$ and $F^{\prime \prime}$. So $Q \cap M^{\prime}$ is a finitely generated $R$-module and $Q \cap F^{\prime}$ is a finite rank projective $R$-module, i.e. $Q \cap F^{\prime}$ is a finitely generated $R$-module implying $Q$ is a finitely generated $R$-module. By Lemma 1.29 of [6], $R=Q$. This completes the proof.

Theorem 1.5(2) does not hold for every semiprime left Goldie ring. Indeed, if $R$ is the subring of $2 \times 2$ matrices over the integers localized at $p$ consisting of matrices $\left(a_{i j}\right)$ with $a_{11} \equiv a_{22}(\bmod p), a_{12} \equiv 0(\bmod p)$, and $a_{21} \in Z_{p}$ for some prime $p$ of $Z$, then $R$ is a local ring and has a simple Artinian quotient ring $Q$. Note that there are rank one torsion free $R$-modules with projective covers. ( $R$ is semiperfect [5].) These $R$-modules are not projective since projective $R$-modules are free. Therefore, an extra hypothesis on the ring $R$ is necessary. The next section gives conditions on a semiprime left Goldie ring which imply $1.5(2)$.

2. Pomo- $C$-algebras and projective covers. For this section $C$ is a Noetherian commutative ring with Artinian quotient ring $K$. Also, $R$ is a $C$-algebra. One says $R$ is finitely generated by $C$ iff $R$ is a finitely generated $C$-module and $R$ is a torsion free $C$-algebra iff $R$ is a torsion free $C$-module. At all times, $C \neq K$. If $C$ is semiprime, then its integral closure in $K$ is hereditary.

Given a multiplicatively closed subset $S$ of the regular elements of $C$ and a $C$-module $X$, let $S^{-1} X=S^{-1} C \otimes_{C} X$ where $S^{-1} C$ is the subring of $K$ generated by $C$ and $\left\{s^{-1}: s \in S\right\}$. Then $S^{-1} C$ is isomorphic to the classical localization of $C$ at $S$.

Definition 2.1. The $C$-algebra $R$ is a pomo- $C$-algebra iff $R$ is a finitely generated torsion free $C$-module.

Note that $C$ may be taken as a subring of the center of $R$. Hence, this identification will be made throughout this section.

A pomo-subring is an order in an Artinian $K$-algebra. To see this, note $R$ may be identified with its image in $K \otimes_{C} R$ since $R$ is a torsion free $C$-module. Since $R$ is finitely generated by $C, K \otimes_{C} R$ is finitely generated by $K$. Thus $K \otimes_{C} R$ is an Artinian $K$-algebra. At all times, we will denote this $K$-algebra by $Q$. Because regular elements of $C$ are regular in $R$, we have $Q$ a quotient ring of $R$. If we identify $R$ with its image in $Q$, then $Q=K R$ : the $K$-submodule of $Q$ generated by $R$. It is then easily shown that $N(R)=N(Q) \cap R$ and $R / N(R)$ is an order in $Q / N(Q)$.

Examples of pomo- $C$-algebras are $C$-orders where $C$ is a Noetherian integral domain (see [13]), and subrings of finite dimensional semisimple algebras over the rationals (see [2]).

Actually, the prefix "pomo" is an abbreviation for "subring of a product of maximal orders." Thus

Proposition 2.2. Let $R$ be a pomo-subalgebra. Then there is a finitely generated C-subalgebra $\Omega$ of $Q / N(Q)$ containing $(R+N(Q)) / N(Q) \cong R / N(R)$ and $\Omega$ is a maximal order in $Q / N(Q)$. 
Proof. It suffices to assume $N(R)=(0)$. By the Wedderburn Theorem, write $Q=Q_{1} \times \cdots \times Q_{t}$ where $Q_{i}$ is a simple Artinian $K$-algebra. Let $e_{i}$ be the central idempotent of $Q$ corresponding to $Q_{i}$ (i.e. $Q e_{i}=Q_{i}$ and $e_{i}$ is an element of $C(Q)$ ). Then $R$ is a subring of the pomo- $C$-algebra $R e_{1} \times \cdots \times R e_{t}$. By convention, $C$ is a subring of $R$, so $C$ is a subring of $C e_{1} \times \cdots \times C e_{t}$ and $C e_{i}$ is a subring of $C\left(Q_{i}\right)$. Thus, each $C e_{i}$ is a Noetherian integral domain. Since $R$ is finitely generated by $C$, it follows that $R e_{i}$ is a $C e_{i}$-order in the simple finite dimensional $K e_{i}$-algebra $Q e_{i}$ (see [13]).

Apply Theorem 10.4 of [13] which states that every such $C e_{i}$-order is contained in a maximal $C e_{i}$-order $\Omega e_{i}$ of $Q_{i}$. Then $\Omega=\Omega e_{1} \times \cdots \times \Omega e_{t}$ is a maximal order in $Q=Q_{1} \times \cdots \times Q_{t}$. Because $Q$ is a $K$-algebra, $\Omega$ is a pomo- $C$-algebra. Finally, $\Omega$ contains $R$ since $\Omega e_{i} \supset R e_{i}$ for $i=1, \ldots, t$. This completes the proof.

Notice that for each finitely generated $C$-module $M$ of $Q$ there is a regular element $c$ of $C$ such that $M \cong c M \subset R$. Also notice that the example following 1.7 is a $Z_{p}$-order. $\left(Z_{p}\right.$ is the integers localized at the prime ideal $p Z$.) Therefore, being a pomo- $C$-algebra does not guarantee that $1.5(2)$ holds. However, the next property is sufficient.

Definition 2.3. Let $R$ be a semiprime pomo- $C$-algebra. Then $R$ has enough primes of $C$ iff there exists a multiplicatively closed subset $S$ of the regular elements of $C$ such that $S^{-1} R \subset Q$ is a semihereditary Noetherian ring, and $S^{-1} J(R) \subset$ $J\left(S^{-1} R\right) .\left(S^{-1} X=S^{-1} C \otimes X\right.$ where $S^{-1} C$ is the usual localization of $C$ at $S$.)

Remark. Assume there exists a maximal order $\Omega$ of $Q$ and a regular element $c$ of $C$ as in 2.2. Further assume there is a prime ideal $I \neq(0)$ of $C$ not containing $c$. Then $R$ has enough primes of $C$ if we set $S$ equal to the regular elements of $C$ not contained in $I$. In this case it is well known that $S$ is multiplicatively closed. Simple computation demonstrates $S^{-1} c \Omega=S^{-1} R=S^{-1} \Omega$, which is semihereditary by [13].

Also note that for this choice of $S, S^{-1} J(R) \subset J\left(S^{-1} R\right)$ since maximal left ideals of $S^{-1} R$ are in one-to-one correspondence with the left ideals of $R / I R$ (see [4]).

THEOREM 2.4. Let $R$ be a semiprime pomo-C-algebra, and say $R$ has enough primes of $C$. Then the finitely generated torsion free $R$-modules with projective covers are the finitely generated projective $R$-modules.

.Proof. Let $M$ be a finitely generated torsion free $R$-module with projective cover $P \stackrel{\pi}{\rightarrow} M \rightarrow 0$. Then $\operatorname{ker} \pi \subset J(R) P$ by Nakayama's Lemma. Let $S$ be the implied collection of regular elements of $C$ and let $S^{-1} P \stackrel{S^{-1} \pi}{\rightarrow} S^{-1} M \rightarrow 0$ be the sequence induced by applying $S^{-1} C \otimes_{C}$ to $P \stackrel{\pi}{\rightarrow} M \rightarrow 0$. By the flatness of $S^{-1} C, \operatorname{ker} S^{-1} \pi=$ $S^{-1}$ ker $\pi$. Hence, $\operatorname{ker} S^{-1} \pi \subset S^{-1} J(R) P \subset J\left(S^{-1} R\right) S^{-1} P$. By Nakayama's Lemma, ker $S^{-1} \pi \ll S^{-1} P$ as $S^{-1} R$-modules. Because tensor product commutes with direct sums, $S^{-1} P$ is a finitely generated projective $S^{-1} R$-module.

Now, $M$ is a torsion free $R$-module.

Claim. $S^{-1} M$ is a torsion free $S^{-1} R$-module. This is clear since the canonical map $M \rightarrow K M$ is a monomorphism and $S^{-1} C$ is a flat $C$-module. Therefore $S^{-1} M$ is a finitely generated torsion free $S^{-1} R$-module. By hypothesis $S^{-1} R$ is a semihereditary 
Noetherian ring. Then $S^{-1} M$ is a projective $S^{-1} R$-module and this shows $S^{-1} \operatorname{ker} \pi=$ (0). Because ker $\pi$ is a torsion free $R$-module, $\operatorname{ker} \pi \subset S^{-1} \operatorname{ker} \pi \subset K \operatorname{ker} \pi$, i.e. $\pi$ is an isomorphism.

This proves the theorem.

Let $I \subset J(T)$ be an ideal of a ring $T$ and assume idempotents lift modulo $I$ (i.e. if $\bar{e}$ is an idempotent of $T / I$, then one may choose an idempotent $e$ of $T$ from the representatives of $\bar{e}$ ). A well-known result states that a finitely generated $T$-module $M$ has a projective cover over $T$ iff $M / I M$ has a projective cover over $T / I$. As a corollary to 2.4 one may deduce more when $T / I$ is a pomo- $C$-algebra.

CoRollary 2.5. Let $T$ be a ring and let $I \subset J(T)$ be an ideal of $T$ such that $T / I$ is a pomo-C-algebra with enough primes of $C$. Assume that idempotents lift modulo I. Let $M$ be a finitely generated T-module such that $M / I M$ is a torsion free $T / I$-module. Then $M$ has a projective cover over $T$ iff $M / I M$ is a projective $T / I$-module.

Proof. Say $M$ possesses a projective cover over $T$ and $M / I M$ is a torsion free $T / I$-module. By the comments preceeding this corollary, $M / I M$ has a projective cover over $T / I$. By $2.4, M / I M$ is a projective $T / I$-module. The converse is a consequence of the aforementioned comments.

3. Properties of quasiprojective covers. This section deals with with some basic properties of quasiprojective covers and the question of their existence over various rings. The basic theme is to show when a quasiprojective cover over $R$ induces a projective cover over a quotient of $R$. Thus, an investigation of quasiprojective modules is in order.

Unless otherwise specified, there are no special assumptions on the ring $R$ or its left module $M$.

Definition 3.1. A left $R$-module $M$ is a singular left module iff $l(m)$ is an essential ideal of $R$ for each $m$ in $M$.

Definition 3.2. A ring $R$ is called left bounded iff every essential left ideal $I$ contains an essential ideal of $R$. A left module $M$ is called bounded iff $l(M)$ is an essential ideal of $R$.

The first result demonstrates quasiprojective covers are more common than projective covers.

Lemma 3.3. Let $R$ be $a$ ring and $M$ a left $R$-module. Then $M$ possesses $a$ quasiprojective cover over $R$ iff $M$ possesses a quasiprojective cover over $R / l(M)$. Moreover, if $M$ possesses a projective cover over $R / l(M)$, then this cover is a quasiprojective cover.

Proof. Let $P \stackrel{\pi}{\rightarrow} M \rightarrow 0$ be a quasiprojective cover of $M$ over $R$. Then $l(M) P \subset$ ker $\pi$ since $\pi(l(M) P)=l(M) M=(0)$. Thus, $P \stackrel{\pi}{\rightarrow} M \rightarrow 0$ is a sequence of $R / l(M)$ modules. (By its definition, the kernel of our cover has no nonzero $\operatorname{End}_{R}(P)$-submodules.) Because $R / l(M)$-modules are $R$-modules, $P$ is a quasiprojective $R / l(M)$ module and ker $\pi$ has no nonzero $\operatorname{End}_{R}(P)$-submodules. Hence, $P \stackrel{\pi}{\rightarrow} M \rightarrow 0$ is a quasiprojective cover over $R / l(M)$. 
Conversely, assume that $P \stackrel{\pi}{\rightarrow} M \rightarrow 0$ is a quasiprojective cover over $R / l(M)$. Again, $R / l(M)$-modules are $R$-modules. Thus an argument as in the above paragraph shows $P \stackrel{\pi}{\rightarrow} M \rightarrow 0$ to be a quasiprojective cover over $R$.

Moreover, assume that $P \stackrel{\pi}{\rightarrow} M \rightarrow 0$ is a projective cover over $R / l(M)$. In [20], it is shown that $M$ possesses a quasiprojective cover and that the quasiprojective is $P / L$ where $L$ is the maximal $\operatorname{End}_{R}(P)$-submodule of ker $\pi$. But then $P \stackrel{\pi}{\rightarrow} P / L \rightarrow 0$ is a projective cover of $P / L$ over $R / l(M)$. Fuller and Hill have shown in [9] that a faithful quasiprojective with a projective cover is a projective module. Thus, $P / L$ is a projective module. This proves $L$ is trivial and so $P \stackrel{\pi}{\rightarrow} M \rightarrow 0$ is a quasiprojective cover.

To demonstrate the ubiquity of quasiprojective covers, we present

COROLlARY 3.4. Let $R$ be a left bounded ring and assume for each essential ideal I of $R$ that $R / I$ is (semi)perfect (e.g. if $R$ has the restricted minimum condition on left ideals). A (finitely generated) bounded left module $M$ has a quasiprojective cover over $R$.

Proof. Since $R$ is left bounded, finitely generated left singular modules are bounded. For if $m_{1}, \ldots, m_{n}$ generate $M$, then $l\left(m_{1}\right) \cap \cdots \cap l\left(m_{n}\right)$ is an essential left ideal of $R$. Let $I$ be an essential ideal of $R$ contained in the intersection. Then $I M=I m_{1}+\cdots+I m_{n}=(0)$.

For (finitely generated) bounded left modules $M, R / l(M)$ is a (semi)perfect ring. Thus $M$ possesses a projective cover over $R / l(M)$. By 3.3 , this cover is a quasiprojective cover over $R$.

Lemma 3.3 gives a sufficient condition on $R$ so that a quasiprojective cover over $R$ is a projective cover over a quotient of $R$. One easily produces necessary conditions for this conclusion by considering the individual sequences.

Proposition 3.5. Let $R$ be any ring and let $P \stackrel{\pi}{\rightarrow} M \rightarrow 0$ be a quasiprojective cover over $R$. Then $P \stackrel{\pi}{\rightarrow} M \rightarrow 0$ is a projective cover over a quotient of $R$ iff $P$ is a projective $R / l(M)$-module.

LEMMA 3.6. Let $P \stackrel{\pi}{\rightarrow} M \rightarrow 0$ be a quasiprojective cover of $M$ over $R$. Then $l(M)=$ $l(P)$.

Proof. Since ker $\pi$ has no nonzero $\operatorname{End}_{R}(P)$-submodules, $l(M) P=(0)$. (l( $\left.M\right) \subset$ ker $\pi$ as shown earlier.) Thus $l(M) \subset l(P)$. The reverse inclusion is clear so the proof is complete.

Proof of 3.5. If $P$ is a projective $R / l(M)$-module, the result follows from 3.3.

If $P \stackrel{\pi}{\rightarrow} M \rightarrow 0$ is a projective cover over $R / I$ for some ideal $I$ of $R$, then $I \subset l(M)=l(P)$ by 3.6. Thus, $P / l(P)=P$ is a projective $R / l(M)$-module.

To show a quasiprojective cover is a projective cover over a quotient of $R$, one must prove that quasiprojective modules are projective modulo their annihilators. Results of this kind are found in [8 and 19]. Thus, if $M$ is finitely generated, then the conclusion of 3.5 holds when $R$ is commutative or a bounded hereditary Noetherian 
prime ring. If $R$ is an order in an Artinian ring, and $M$ is finitely generated and torsion free, the conclusion to 3.5 is true.

Finally,

LEMMA 3.7. Let $P \stackrel{\pi}{\rightarrow} M \rightarrow 0$ be a quasiprojective cover and say there is a finite subset $X$ of $M$ such that $l(X)=l(M)$. Then $P$ is a projective $R / l(M)$-module if $M$ is finitely generated.

Proof. For $x$ in $X$ let $f_{x}: R \rightarrow M$ be $f_{x}(r)=r x$. Because $l(X)=l(M)$, the map $f: R \rightarrow M^{(X)}$ defined by $f(r)=\left(f_{x}(r), \ldots, f_{x}(r)\right)$ has kernel $l(M)$. Hence, $R / l(M)$ is embedded in $M^{(X)}$. It is easily seen that $R / l(M)$ is then embedded in $P^{(X)}$ by the projectivity of $R / l(M)$. Then $(0.2)$ shows that $P^{(X)}$ is a projective $R / l(M)$-module. This concludes the proof.

The remainder of the paper is devoted to the investigation of quasiprojective covers over pomo- $C$-algebras. Thus $R$ will be a pomo- $C$-algebra and $Q$ will denote the quotient ring of $R$. Note $Q$ is an Artinian ring. The first results are on the structure of torsion free quasiprojective $R$-modules. From these are derived results on quasiprojective covers over $R$.

If $R$ is a semiprime pomo- $C$-algebra, then $\Omega$ will denote a maximal order in $Q$ containing $R$. An $R$-module is reduced iff it has no nonzero injective summands.

LEMMA 3.8. Let $R$ be a semiprime pomo-C-algebra and let $M$ be a reduced torsion free quasiprojective $R$-module. Then $\Omega M$ (the $\Omega$-module generated by $M$ in $Q M$ ) is a projective $\Omega$-module.

Proof. Let $c$ be a regular element of $C$ such that $c \Omega \subset R$.

Claim. $\Omega M$ is a quasiprojective $\Omega$-module. The argument requires a rudimentary understanding of relative projectivity.

Since $c \Omega \subset R, c \Omega M \subset M$. Therefore, $M$ is a $c \Omega M$ projective module (0.2). Because $M$ is torsion free, $c \Omega M \cong \Omega M$. Let $\Omega M \stackrel{\pi}{\rightarrow} X \rightarrow 0$ be an epimorphism of $\Omega$-modules, and let $f: \Omega M \rightarrow X$ be any $\Omega$-module homomorphism. Then $f: M \rightarrow X$ is an $R$-module map as is $\pi$. By relative projectivity, there is an $R$-module map $g$ : $M \rightarrow \Omega M$ such that $\pi \circ g=g$. But $g$ extends linearly to an $\Omega$-module homomorphism $h: \Omega M \rightarrow \Omega M$. Then $\pi \circ h=f$ since $f$ and $\pi \circ h$ agree on the generators of $\Omega M$, i.e. $\Omega M$ is a quasiprojective $\Omega$-module as claimed.

Now $\Omega M$ is reduced since $M$ is reduced. Then an application of [19] shows $\Omega M$ is a projective $\Omega$-module. ([19] shows that the reduced torsion free quasiprojectives over a hereditary Noetherian semiprime ring are projective modulo their annihilators. Maximal orders are known to fit these hypotheses.)

THEOREM 3.9. Let $R$ be a prime pomo-C-algebra, and let $M$ be a nontorsion reduced $R$-module. Then $M$ is a quasiprojective $R$-module iff $M$ is a projective $R$-module.

Proof. By Theorem 2 of [19], $M$ is torsion free.

Say $M$ is a quasiprojective $R$-module. Let $c$ and $\Omega$ be as in 3.8. By 3.8, $\Omega M$ is a projective $\Omega$-module. If $M$ has finite rank then $\Omega M$ is a finitely generated projective $R$-module. ( $\Omega$ is finitely generated by $C$.) Then $M$ is a finitely generated quasiprojective torsion free $R$-module. By [19], $M$ is a projective $R$-module. 
If $M$ has infinite rank, then $\Omega M$ is a free $\Omega$-module by [7]. Say $M$ has rank $r$ for some infinite cardinal $r$. Now $\Omega M \supset M \supset c \Omega M$ and $c \Omega M$ contains a free $R$-module $F$ on $r$ elements. Assume the basis for $F$ is a basis of $\Omega M$. Then $\Omega M \supset M \supset F$. Because $\Omega / R$ is a finitely generated $R$-module, and because $\Omega M / F$ is the direct sum of $r$ copies of $\Omega / R, M$ is generated by fewer than $r+\aleph_{0} r=r$ elements. $(M / F \subset$ $\Omega M / F$ and $\Omega M / F$ is generated by $\kappa_{0} r$ elements. $R$ is Noetherian so $M / F$ is generated by at most $\boldsymbol{\aleph}_{0} \boldsymbol{\aleph}_{0} r=r$ elements.) By $(0.2), M$ is $F$-projective and by the freeness of $F$, there is an epimorphism $f: F \rightarrow M$. By relative projectivity, $f$ splits. Thus $M$ is a projective $R$-module. The converse is clear so the proof is complete.

COROLlARY 3.10. Let $R$ be a semiprime pomo-C-algebra, and let $M$ be a torsion free reduced $R$-module. Then $M$ is a quasiprojective $R$-module iff $M$ is a projective $R / l(M)$-module.

Proof. First, reduce to the case where $M$ is a faithful $R$-module. To do this, it suffices to prove that $R / l(M)$ is a pomo- $C$-algebra. Since $K$ is central in $K R, K l(M)$ is an ideal of $K R$. Thus, there is a ring homomorphism from $R$ into $K R / K l(M)$. Then $\bar{R}=R /[R \cap K l(M)]$ is a subring of $K R / K l(M)$. Note $\bar{R}$ is a pomo-C-algebra with quotient ring $K \bar{R}=K R / K l(M)$. But $l(M) \subset K l(M)$ and for an element $x$ of $R \cap K l(M), x M \subset x K M=(0),(M$ is torsion free). Thus $x$ is an element of $l(M)$ implying $l(M)=R \cap K l(M)$, i.e. $\bar{R}=R / l(M)$. Hence $R / l(M)$ is a pomo- $C$ algebra and $M$ is a faithful torsion free quasiprojective $R / l(M)$-module.

Assume that $M$ is a faithful torsion free quasiprojective module. By the Wedderburn Theorem, write $Q=Q_{1} \times \cdots \times Q_{t}$, where $Q_{i}$ is a simple Artinian ring. Let $e_{i}$ be the central idempotent of $Q$ corresponding to $Q_{i}$. Then $R$ is a subring of $R e_{1} \times \cdots \times R e_{t}$, and $R e_{i}$ is an order in $Q_{i}$. Considering $M$ as a submodule of $Q M$, one has $M$ a submodule of $e_{1} M \oplus \cdots \oplus e_{t} M$, and each $e_{i} M$ is a reduced nonzero torsion free $R e_{i}$-module.

Let $L_{i}=l\left(R e_{i}\right)$ be an ideal of $R$. Then $L_{i} e_{i} M=(0)$ so there is an $R$-module epimorphism from $M / L_{i} M$ onto $e_{i} M$. Since $e_{i} M$ is reduced and torsion free, $M / L_{i} M$ is a reduced nontorsion $R / L_{i}=e_{i} R$-module. By Lemma 4 of [20], $M / L_{i} M$ is a nontorsion quasiprojective $e_{i} R$-module. By 3.9, $M / L_{i} M$ is a projective $e_{i} R$-module. Thus for each cardinal $r, M / L_{i} M$ is an $e_{i} R^{(r)}$-projective $R$-module. Furthermore, for $e_{i} R^{(r)} \rightarrow X \rightarrow 0$ and $R$-module homomorphisms $g: M \rightarrow X$, $\operatorname{ker} g \supset L_{i} M$. Therefore $M$ is $e_{i} R^{(r)}$-projective for each cardinal $r$. ( $g$ induces a map from $M / L_{i} M$ into $X$ and this map lifts to a map $h: M / L_{i} M \rightarrow e_{i} R^{(r)}$.) By (0.2), $M$ is projective relative to $e_{1} R^{(r)} \oplus \cdots \oplus e_{t} R^{(r)}$ for each cardinal $r$. Because $R$ is a submodule of $e_{1} R \oplus \cdots \oplus e_{t} R, M$ is an $R^{(r)}$-projective $R$-module for each cardinal $r$. Because $M$ is the image of a free $R$-module, $M$ is a projective $R$-module. $\left(R^{(r)} \rightarrow M \rightarrow 0\right.$ is split exact for some $r$ by relative projectivity.)

Since 3.10 shows that the faithful torsion free reduced quasiprojective modules are projective, one is immediately led to results on quasiprojective covers.

COROLlaRY 3.11. Let $R$ be a semiprime pomo-C-algebra, and let $P \stackrel{\pi}{\rightarrow} M \rightarrow 0$ be $a$ quasiprojective cover over $R$. If $M$ is torsion free reduced, then $P \stackrel{\pi}{\rightarrow} M \rightarrow 0$ is $a$ projective cover over $R / l(M)$. 
Proof. If $M$ is torsion free reduced, then $P$ is torsion free reduced. (The torsion submodule and injective submodule of $P$ are in ker $\pi$.) 3.10 and 3.5 then apply.

COROLlARY 3.12. If $R$ is a semiprime pomo-C-algebra, and has enough primes of $C$, then a quasiprojective cover of a reduced torsion free $R$-module is an isomorphism.

Proof. Apply 3.5 and 3.11 .

COROLlaRY 3.13. Let $P \stackrel{\pi}{\rightarrow} M \rightarrow 0$ be a quasiprojective cover of $M$ over $R$. If $P$ is a torsion free reduced $R$-module, then $t(M)$ is finitely generated.

Proof. Apply 3.11 and 1.3.

Corollary 3.14. Say $R$ is a semiprime pomo-C-algebra and let $M$ have a quasiprojective cover over $R$. If $M$ is torsion free reduced of finite rank, then $M$ is finitely generated by $R$.

Proof. Apply 3.11 and 1.4.

COROllary 3.15. Let $R$ be a semiprime pomo-C-algebra. The following are equivalent:

(1) $R$ is left hereditary.

(2) Submodules of projective $R$-modules have quasiprojective covers.

(3) Left ideals of $R$ are quasiprojective.

PROOF. $1 \Rightarrow 2$ is clear since submodules of projective modules are hereditary.

$2 \Rightarrow 1$. Let $M$ be a submodule of a projective module. Then $M \oplus R$ is a faithful submodule of a projective $R$-module and so has a faithful quasiprojective cover. Let $P$ be the quasiprojective. Then $P$ is a faithful quasiprojective $R$-module (3.6) and by 3.10 , and 3.5, $P$ is a projective module. Thus $M$ has a projective cover over $R$. Now apply 1.6 .

$1 \Rightarrow 3$ is clear since the left ideals of $R$ are projective.

$3 \Rightarrow 1$. In [9], it is shown that if $I$ is a finitely generated quasiprojective left $R$-module, then $I^{(r)}$ is a quasiprojective left $R$-module for each cardinal $r$. Now apply 1.6 .

COROllaRY 3.16. Let $R$ be a semiprime pomo-C-algebra with enough primes of $C$. Let $M$ be a torsion free reduced $R$-module. Then $M$ has a quasiprojective cover over $R$ iff $M$ is a projective $R / l(M)$-module.

Proof. If $M$ has a quasiprojective cover, then 3.11 shows $M$ has a projective cover over $R / l(M)$. Apply 2.4 to derive the required result. The converse is clear so the proof is complete.

Finally, in order to demonstrate the necessity of the hypotheses in $\S 3$, we present a few examples.

EXAMPLE 1. In [12], it is shown that the $p$-adic numbers $\bar{Q}_{p}$ form a quasiprojective module over the $p$-adic integers $\bar{Z}_{p}$. Since $\bar{Z}_{p} \ll \bar{Q}_{p}$ one constructs a quasiprojective cover $\bar{Q}_{p} \rightarrow \bar{Q}_{p} / \bar{Z}_{p} \rightarrow 0$. Note that $\bar{Q}_{p}$ is not a projective $\bar{Z}_{p}$-module nor is the torsion module $\bar{Q}_{p} / \bar{Z}_{p}$ a finitely generated $\bar{Z}_{p}$-module. Compare this to $3.10-3.15$. 
EXAMPLE 2. The group $\oplus_{p} Z / p Z$ is a quasiprojective $Z$-module as noted in [12]. Note that this group is a singular (torsion) $Z$-module, but is not projective modulo its trivial annihilator. Compare to 3.4.

EXAMPLE 3. In [19], it was demonstrated that $Z / p Z \oplus Z / p^{2} Z=M$ is not a quasiprojective $Z$-module and since $J(Z)=(0), M$ does not have a projective cover over $Z$. However, $M$ has a quasiprojective cover given by $Z / p^{2} Z \oplus Z / p^{2} Z \rightarrow M \rightarrow 0$. This shows that finitely generated nonquasiprojective modules may have quasiprojective covers. Also, compare to 3.3, 3.4, and 3.6.

EXAmple 4. $\bigoplus_{k} Z / p^{k} Z=M$ is a singular (torsion) $Z$-module. but does not have a quasiprojective cover over $Z$. This is seen by noting $M$ is a faithful module. Then [19] and 3.6 combine to show the quasiprojective cover is a projective cover. Since $J(Z)=(0)$, this cover is an isomorphism. This contradiction proves $M$ is without a quasiprojective cover.

I wish to thank Dr. Charles Vinsonhaler for his guidance during the construction of my dissertation of which this paper forms a part.

\section{REFERENCES}

I. F. W. Anderson and K. R. Fuller. Rings and categorles of modules. Springer. Berlin and New York . 1974.

2. D. Arnold. Torsion free abelian groups of finite rank and subrings of finite dimensional Q-algebras. Lecture Notes in Math., vol. 931. Springer-Verlag. Berlin and New York. 1982.

3. D. Arnold, R. Pierce, J. Reid, C. Vinsonhaler and W. Wickless, Torsion free abelian groups of finite rank projective as modules over their endomorphism rings. J. Algebra (to appear).

4. M. F. Atiyah and I. G. McDonald, Introduction to commutative algehra. Addison-Wesley, Reading. Mass., 1979.

5. H. Bass, Finitistic dimension and a homological generalization of semi-primary rings. Trans. Amer. Math. Soc. 95 (1960), 466-488.

6. A. W. Chatters and C. R. Hajarnavis, Rings with chain conditions, Pitman, Boston. Mass., 1980

7. D. Eisenbud and J. C. Robson, Modules over Dedekind prime rings, Algebra 16 (1970), 67-85.

8. T. G. Faticoni, On classifying quasi-projective modules, Finitely faithful modules and finitely gencrated quasi-projective modules, Comm. Algebra (submitted).

9. K. R. Fuller and D. A. Hill, On quasi-projective modules via relative projectivity, Arch. Math. (Basel) 21 (1970), 369-373

10. J. Lambek, Lectures on rings and modules, Blaisdell, Waltham, Mass., 1966.

11. K. M. Rangaswamy, Some remarks on the endomorphism rings of quasi-projective modules (to appear).

12. K. Rangaswamy and N. Vanja, Quasi-projectives in abelian and module categories, Pacific J. Math. 43 (1972), 221-238.

13. I. Reiner, Maximal orders, Academic Press, New York, 1975.

14. K. W. Roggenkamp and V. H. Dyson, Lattices over orders. I, Lecture Notes in Math., vol. 115 , Springer-Verlag. Berlin and New York, 1970.

15. K. W. Roggenkamp, Lattices over orders. II, Lecture Notes in Math., vol. 142, Springer-Verlag, Berlin and New York, 1970.

16. S. Singh, Quasi-injective and quasi-projective modules over hereditary Noetherian prime rings, Canad. J. Math 26 (1974), 1173-1185.

17. __ Modules over hereditary Noetherian prime rings, Canad. J. Math. 27 (1975), 867-883

18. B. Stenstrōm, An introduction to methods of ring theory, Grundlehren Math. Wiss., Bd. 217, Springer-Verlag, Berlin and New York, 1975.

19. A. A. Tuganbaev, Quasiprojective modules, Sibirsk Mat. Z. (3) 21 (1980), 177-183, 238.

20. L. E. T. Wu and J. P. Jans, On quasi-projectives, Illinois J. Math. 11 (1967), 439-448.

Department of Mathematics, New Mexico State University, Las Cruces, New Mexico 88003

Current address: Department of Mathematics, University of Lowell, Lowell, Massachusetts 01854 